\title{
A minor subset of HLA-DR3 haplotypes is preferentially increased in Type 1 (insulin-dependent) diabetes
}

\author{
M.J.Sheehy ${ }^{1,2}$, J.R. Rowe ${ }^{2}$, T.C. Fuller ${ }^{5}$, E.J. Yunis ${ }^{4,5}$ and K.H.Gabbay ${ }^{3,6 *}$ \\ ${ }^{1}$ Section of Endocrinology, Department of Medicine, University of Wisconsin and ${ }^{2}$ American Red Cross Blood Services, Madison, Wisconsin; \\ ${ }^{3}$ Diabetes Unit, Children's Hospital Medical Center; ${ }^{4}$ Division of Immunogenetics, Dana/Farber Cancer Institute and Departments of \\ ${ }^{5}$ Pathology and ${ }^{6}$ Pediatrics, Harvard Medical School, Boston, Massachusetts, USA
}

\begin{abstract}
Summary. We have been using human T-lymphocyte clones specifically sensitized to detect leucocyte antigens of Type 1 (insulin-dependent) diabetic patients in the hope of detecting novel HLA antigens associated with Type 1 diabetes. We previously described two such clones which define a new class II HLA antigen, Boston-1 (BO1). BO1 is found mainly on cells of persons with particular HLA-DR antigens and, of potential significance for diabetes, $\mathrm{BO} 1$ identifies a distinctive subset of $\mathrm{DR} 3$ haplotypes. We report here that $\mathrm{BO}^{+} \mathrm{DR} 3$ haplotypes
\end{abstract}

are overrepresented in Type 1 diabetes. That is, significantly more of the DR3-positive subjects are BO1-positive in the patient group (31\%) than in the control group (8\%), suggesting that a diabetes-susceptibility gene may be more common on the $\mathrm{BO}^{+}$than on the $\mathrm{BO} 1^{-} \mathrm{DR} 3$ haplotypes. Alternative interpretations are also discussed.

Key words: Type 1 (insulin-dependent) diabetes, genetics, HLA antigens, T-lymphocyte clones.
Type 1 (insulin-dependent) diabetes mellitus is determined in part by genes in the HLA region of human chromosome 6 [1-6]. Although in most studies the strongest associations are with HLA-DR3 and 4, not all Type 1 diabetic patients have DR3 or 4 ; this suggests that these antigens may merely be markers for nearby genes truly involved in the disease process.

To better define the HLA gene products involved in Type 1 diabetes, we are systematically producing reagents detecting leucocyte antigens of Type 1 diabetic patients. These reagents are human T-lymphocyte clones or oligoclonal colonies derived from mixed leucocyte cultures with "responder" cells from a non-diabetic person and gamma-irradiated "stimulator" cells from a Type 1 diabetic patient. Such clones or colonies, detecting various leucocyte antigens possessed by the patient but not by the non-diabetic responder, are used in a proliferative assay called primed lymphocyte typing or PLT [7].

We described a new class II HLA antigen, Boston-1 (BO1) [8], detected by two such T-cells colonies derived from a mixed leucocyte culture in which both the nondiabetic responder and the diabetic stimulator were HLA-DR3/4 heterozygotes. Among random controls, BO1 was most strongly associated with HLA-DR5 (23/23 DR5-positive subjects being BO1-positive), but

* Current address: Department of Pediatrics, Baylor College of Medicine, Houston, Texas 77030 , USA it also reacted with cells of about half (5 of 9) of DRw6-positive subjects, a small subset of DR3-positive subjects (the subject of this paper), and a few subjects with none of these antigens.

To determine the possible relationships of the BO1-positive and BO1-negative DR3 subsets to Type 1 diabetes, we determined the frequency of the $\mathrm{BO} 1$ antigen among DR3-positive Type 1 diabetic patients and non-diabetic controls as described here.

\section{Subjects and methods}

\section{Subjects}

Patients (25 females and 35 males) were Caucasians with classical ketosis-prone Type 1 (insulin-dependent) diabetes diagnosed before age 15. All were followed in the Diabetes Control and Vascular Disease Study at Children's Hospital in Boston. The 39 controls were healthy adults living or working in Boston. No attempt was made to use controls age- or sex-matched with the patients, since HLA antigens are genetically determined and there is no evidence that they are correlated with age or sex in healthy individuals.

Informed consent was obtained for all subjects, and this research was approved by Human Subjects Committees at the authors' respective institutions.

\section{Methods}

HLA typing was done by the standard National Institutes of Health microcytotoxicity technique [9].

Production and maintenance of human T-cell colonies B33-25 and B33-30 were done by widely used techniques as described earlier [8]. 


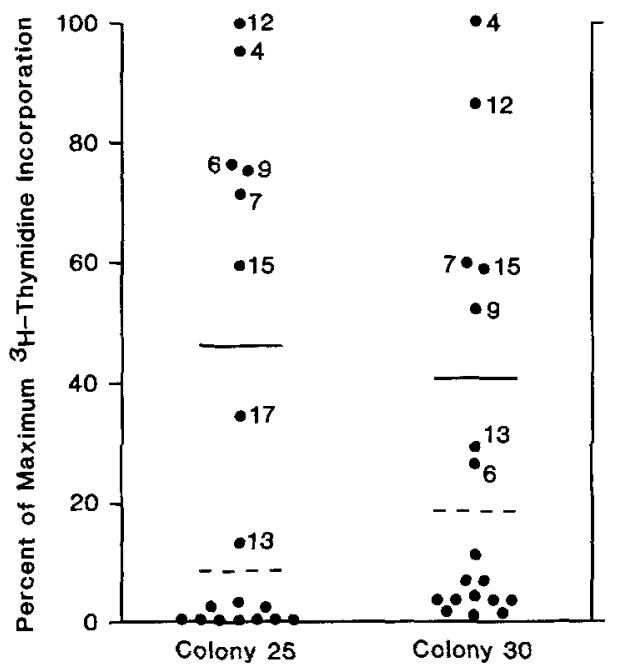

Fig.1. Example of a primed lymphocyte typing (PLT) experiment testing 18 subjects for the $\mathrm{BO} 1$ antigen. Each point represents the proliferative response (cpm from ${ }^{3} \mathrm{H}$-thymidine incorporation) of either Colony 25 or 30 to cells of a single subject. Numbers at individual points are subject numbers (shown for positive and intermediate responses only). Raw data are median cpm of triplicate cultures; to facilitate presentation in a single figure, results for each T-cell colony are expressed as a percent of the highest response of that colony. In this experiment, maximum responses of Colonies 25 and 30 were $28,380 \mathrm{cpm}$ and $8,816 \mathrm{cpm}$, respectively. For each colony, responses were divided into "positive" (above solid line), "intermediate" (between broken and solid lines), and "negative" (below broken line) clusters [11]. Individuals causing positive responses by both colonies, or one positive and one intermediate response, are labelled BO1-positive; all others are BO1-negative

Table 1. Increased frequency of antigen BO1 among DR3-positive Type 1 diabetic patients versus DR3-positive controls

\begin{tabular}{llll}
\hline & $\begin{array}{l}\text { Number of } \\
\mathrm{DR}^{+} \mathrm{BO}^{+}\end{array}$ & $\begin{array}{l}\text { Number of } \\
\mathrm{DR3}^{+} \mathrm{BO} 1^{-}\end{array}$ & Percent BO1 $^{+}$ \\
\hline Patients & 14 & 31 & $\begin{array}{c}31.1 \% \\
p=0.029^{\mathrm{a}} \\
\text { Controls }\end{array}$ \\
\hline
\end{tabular}

DR3/5 heterozygotes were excluded for reasons explained in the text. a $p$ value determined by the Fisher Exact Test [12]

Table 2. Negative and positive associations of $\mathrm{BO} 1$ with a northern European DR3 haplotype, B8-SC01-DR3, a and a southern European haplotype, B18-F1C30-DR3, respectively

\begin{tabular}{lll}
\hline $\begin{array}{l}\text { Patients' } \\
\text { DR3 haplotypes: }\end{array}$ & $\begin{array}{l}\text { Number of } \\
\text { BOI-positive }\end{array}$ & $\begin{array}{l}\text { Number of } \\
\text { BOI-negative }\end{array}$ \\
\hline 1. B8-SC01-DR3 & 0 & 18 \\
2. B18-F1C30-DR3 & 6 & 0 \\
3. Other DR3 haplotypes & 7 & 7 \\
4. 1 and 3 & 1 & 1
\end{tabular}

a $\mathrm{SC} 01=\mathrm{BfS}, \mathrm{C} 2 \mathrm{C}, \mathrm{C} 4 \mathrm{~A} 0, \mathrm{C} 4 \mathrm{~B} 1{ }^{\mathrm{b}} \mathrm{F} 1 \mathrm{C} 30=\mathrm{BfF} 1, \mathrm{C} 2 \mathrm{C}, \mathrm{C} 4 \mathrm{~A} 3$, C4B0. Patients are listed according to whether they have the B8-bearing "extended" DR3 haplotype, the B18-bearing extended haplotype, or other DR3 haplotypes. Two patients (line 4) each have the B8 extended haplotype plus another DR3 haplotype
Briefly, peripheral blood mononuclear cells from a non-diabetic person were stimulated in mixed leucocyte culture for 6 days with gamma-irradiated peripheral blood mononuclear cells of a Type 1 diabetic patient to select for lymphocytes of the non-diabetic person that were reactive with HLA antigens of the patient. Cells present on day 6 were enriched for activated blast cells by centrifugation on a discontinuous density gradient, and the blast-rich fraction was "cloned" at an average of 1 cell/well with interleukin-2 (T-cell growth factor) and gamma-irradiated feeder cells. Since colonies grew in $32 \%$ of wells, one calculates from the Poisson distribution that $82 \%$ of colonies should be true clones with the remainder being oligoclonal. Thus, Colonies B33-25 and -30 are likely to be true clones. Both colonies were stored as frozen aliquots.

Primed lymphocyte typing [7], done as described previously [8], is a cells proliferation assay in which in vitro-sensitized (primed) lymphocytes are used to detect HLA antigen(s) to which they have been sensitized. T-cell colonies B33-25 and $-30\left(5-20 \cdot 10^{3} /\right.$ well $)$ were tested in triplicate cultures with peripheral blood mononuclear cells of each subject $\left(5 \cdot 10^{4} /\right.$ well). Cultures, in round-bottom wells of 96 -well culture plates, were pulsed with ${ }^{3} \mathrm{H}$-thymidine from hours 48-64 and harvested onto glass-fiber filters for liquid scintillation counting. Strong proliferation (high $\mathrm{cpm}$ ) indicates that the particular subject has the antigen recognized by the particular $T$-cell colony.

Primed lymphocyte typing results were classified as positive, negative, or intermediate by a least-squares cluster analysis [10] as modified by Shaw et al. [11]. A subject was considered BO1-positive if his/ her cells gave positive stimulation of either Colony B33-25 or -30, and positive or intermediate stimulation of the other. This is illustrated in Figure 1. As defined by the cluster analysis, Colony 25 gave "positive" proliferative responses to cells of subjects $4,6,7,9,12$, and 15 ; "intermediate" responses to cells of subjects 13 and 17; and "negative" responses to the remaining subjects' cells. Responses of Colony 30 were similar but not identical. Cells causing positive responses for both Colonies 25 and 30 , or one positive and one intermediate response, were defined as BO1-positive. Thus individuals $4,6,7,9,12$, and 15 are BO1-positive while the remaining twelve subjects are BO1-negative.

Relative risk $[12,13]$ for any antigen is an odds-ratio: (number of patients with the antigen/number of patients without the antigen) / (number of controls with the antigen/number of controls without the antigen).

\section{Study design}

The study was done in two stages. In a preliminary phase, $18 \mathrm{~T}$-cell colonies were used for primed lymphocyte typing of leucocytes from 15 Type 1 diabetic patients and 15 non-diabetic controls, the control group being pairwise HLA-DR-matched with the patients. Two colonies (B33-25 and -30) detecting an antigen that we refer to as BO1 [8] were identified as potentially interesting since they appeared to detect an HLA-DR3 subset selectively increased in the patient group. Because these two colonies were selected retrospectively after looking at primed lymphocyte typing data from 18 colonies, these data were not included in the statistical analysis.

In a prospective phase, all subjects ( 45 patients and 24 controls) were selected to be HLA-DR3-positive, and did not include the original 15 patients and 15 controls. DR3/5 heterozygotes were excluded; since all $\mathrm{DR} 5^{+}$individuals tested have been $\mathrm{BO}^{+}[8]$, the presence of DR5 would obscure a possible association of $\mathrm{BO} 1$ with a subset of DR3. (Half of DRw6-positive individuals have been BO1-positive as well [8], but there were no DR3/w6 heterozygotes in our subject populations; thus DRw6 was not a potential confounding factor.) These DR3-positive individuals (DR3/5 heterozygotes excluded) were tested for antigen $\mathrm{BO} 1$ by primed lymphocyte typing as described above.

In addition, because of earlier results [8] indicating a probable association of BO1 with an HLA haplotype B18, BfF1, C2C, C4A3, C4BO, DR3 (B18-F1C30-DR3), common in southern Europe and described by others [5], we tested the relationship of $\mathrm{BO} 1$ to this haplotype (the latter defined by HLA typing and by electrophoretic testing of properdin factor $\mathrm{B}(\mathrm{Bf})$, and the second (C2) and fourth (C4A and C4B) components of complement). 
Results

\section{Preliminary studies}

Preliminary testing of pairwise HLA-matched Type 1 diabetic and control groups indicated an excess of the newly-defined BO1 antigen in the patient group. Subjects of this preliminary study were 15 patients and 15 controls, including, in each group: $7 \mathrm{DR} 3 / 4$, $1 \mathrm{DR} 1 / 3,1 \mathrm{DR} 3 / 7,2 \mathrm{DR} 3$ only, $1 \mathrm{DR} 1 / 4,1 \mathrm{DR} 4 / 7$, and 2 DR 4 only. Of these, 6 patients ( $40 \%$ ) and 1 control (7\%) were BO1-positive, and BO1 appeared to be a subtype of DR3. Thus, we conducted a prospective study to determine whether BO1 marks a distinctive DR3 subset selectively increased in Type 1 diabetes.

\section{Prospective study}

Subjects for the prospective phase were tested in groups of 12-24 for antigen BO1 by primed lymphocyte typing (PLT). Results are summarized in Table 1: 31\% (14/45) of DR3-positive patients versus only $8 \%(2 / 24)$ of DR3-positive controls were $\mathrm{BO} 1^{+}(p<0.03)$. Because this was the only statistical test performed (the exact comparison to be tested, including the exclusion of DR3/ 5 heterozygotes, having been decided before commencing the study), there was no need to correct for multiple possible comparisons. In the population tested, therefore, there is an excess of $\mathrm{DR}^{+}{ }^{+} \mathrm{BO} 1^{+}$versus $\mathrm{DR}^{+}{ }^{+} \mathrm{BO} 1^{-}$individuals in the patient group.

In our random patient and control samples, $60 \%$ of patients $(47 / 78)$ and $29 \%$ of controls $(14 / 48)$ were DR3-positive, for a relative risk of 3.7. Using these figures, and the frequencies of BO1 in DR3-positive patients and controls from Table 1 , one calculates a relative risk of 9.3 for the $\mathrm{DR} 3^{+} \mathrm{BO} 1^{+}$subset and 1.9 for the $\mathrm{DR}^{+}{ }^{+} \mathrm{BO} 1^{-}$subset. Thus, besides the increased risk of Type 1 diabetes for DR3-positive persons, reported many times, we find an additional increase for persons with the BO1-positive DR3 subset.

\section{Characterization of the BO1-positive DR3 subset}

Table 2 shows that BO1 is strongly associated with a southern European DR3 haplotype B18-F1C30-DR3 (defined in Subjects and Methods): 6 of 6 patients with that haplotype are BO1-positive, while 0 of 18 with the haplotype B8-SC01-DR3, common in northern Europe, are BO1-positive. BO1 is not universally associated with the B18-F1C30-DR3 haplotype, however; persons with other less common DR3 haplotypes (Table 2, lines 3 and 4) are often BO1-positive. Moreover, the eight BO1-positive individuals in lines 3 and 4 of Table 2 have DR3 haplotypes with neither B8 nor B18 nor the sets of complement alleles (F1C30 or SC01) listed above. Thus BO1 subdivides the DR3 haplotypes in a unique way.

\section{Discussion}

Although several reports indicate a preferential increase of certain DR4 haplotypes in Type 1 (insulin-dependent) diabetes (15-18), there has been little information on possible heterogeneity of DR3 with regard to diabetes susceptibility. The current study and that of Raum et al. [19] show, by two very different techniques, that certain DR3 haplotypes are overrepresented in DR3-positive Type 1 diabetic patients versus DR3-positive controls. It is possible, therefore, that neither DR3 nor DR4 is a primary risk factor for Type 1 diabetes.

We show here that a minor subset of DR3 haplotypes, bearing the recently-defined class II HLA antigen BO1 [8], is selectively increased in Type 1 diabetes. This suggests that the $\mathrm{BO} 1$-positive DR3 subset (relative risk 9.3) is more diabetogenic than the BO1-negative DR3 subset (relative risk 1.9). This could occur either if (a) the DR3-diabetes association is not due to DR3 itself but rather to a still-undefined diabetes-susceptibility gene, with the latter being more common on $\mathrm{BO} 1^{+}$than on $\mathrm{BO} 1^{-} \mathrm{DR} 3$ haplotypes; or (b) a gene on the $\mathrm{BO} 1^{+}$ DR3 haplotypes acts synergistically with DR3 in producing diabetes.

Alternatively, the various DR3 haplotypes may be equally diabetogenic, but the BO1-positive haplotypes may be more common in ethnic groups with other genetic or environmental risk factors. We are not aware of genetic or epidemiologic data that would make it possible to distinguish this possibility from that discussed in the preceding paragraph.

Finally, the sampling procedure must be considered. Patients were from the Diabetes Unit at Children's Hospital Medical Center in Boston, and controls were Boston hospital or blood bank employees $(n=14)$, medical students $(n=4)$, and platelet pheresis donors $(n=6)$. It is possible that this sampling procedure resulted in an ethnic bias, although we have no reason to believe that this should have been the case. Unfortunately, comparing the ethnic mix of patient and control groups would not be informative regarding the possibility of sampling bias, since all three hypotheses would predict a relative excess of southern European-derived persons in the patient group. For example, if a "southern European" DR3 haplotype is more diabetogenic than the other DR3 haplotypes, even an unbiased sampling procedure would produce a relative excess of patients of southern European background.

Like any such study, this one must be replicated by other research groups, particularly in other ethnically mixed populations. Our Colonies B33-25 and -30 are available for such studies, and primed lymphocytes of Termijtelen et al. [22] define an antigen, LB-Q1 [22], that may be equivalent to BO1 (A.Termijtelen, personal communication).

As mentioned above, our results suggest the possibility that the DR3-diabetes association is due to a stillundefined HLA factor. We are continuing the studies 
described here in the hope of producing additional Tcell clones that better define the DR3- and/or DR4-associated diabetes-susceptibility factors.

Acknowledgements. We thank the diabetic patients and their families for voluntary blood donations, Ms. S Schwartz for drawing the blood samples, and Dr. C Alper and associates for characterizing the complement alleles. This work was supported in part by grants from the Juvenile Diabetes Foundation and the Wisconsin Affiliate of the American Diabetes Association, by a Career Development Award from the Juvenile Diabetes Foundation (to MJS), by NIH grants CA20531, HL-34999 (HL-20539), and AM-34504, and by a General Research Support Grant to the University of Wisconsin Medical School from the National Institutes of Health, Division of Research Facilities and Resources. MJS is an Established Investigator of the American Red Cross.

\section{References}

1. Nerup J, Platz P, Anderson OO, Christy M, Lyngsøe J, Poulsen JE, Ryder LP, Thomsen M, Nielsen LS, Svejgaard A (1974) HL-A antigens and diabetes mellitus. Lancet 2: 864-866

2. Garovoy MR, Barbosa J, Yunis E, Carpenter CB (1978) HLA-DR antigens and unique serologic reactions in juvenile-onset diabetes mellitus. Transplant Proc 10:967-969

3. Christy M, Green A, Cristau B, Kromann H, Nerup J, Platz P, Thomsen M, Ryder LP, Svejgaard A (1979) Studies of the HLA system and insulin-dependent diabetes. Diabetes Care 2: 209-214

4. Raum D, Alper CA, Stein R, Gabbay KH (1979) Genetic marker for insulin-dependent diabetes mellitus. Lancet 1: 1208-1210

5. deMouzon A, Ohayon E, Ducos J, Hauptman G (1979) Bf and C4 markers for insulin-dependent diabetes in Basques. Lancet 2: 1364

6. Bertrams J, Dewald G, Spitznas M, Rittner Ch (1980) HLA-A, B, $\mathrm{C}, \mathrm{DR}, \mathrm{Bf}$, and $\mathrm{C} 2$ alleles in insulin-dependent diabetes mellitus with proliferative retinopathy. Immunobiology 158: 113-118

7. Sheehy MJ, Sondel PM, Bach ML, Wank R, Bach FH (1975) HL-A LD typing: A rapid assay using primed lymphocytes. Science 188: $1308-1310$

8. Sheehy MJ, Quinteri FB, Yang SY, Alosco SM, Matsui Y, Yunis EJ, Gabbay KH (1984) HLA antigens of insulin-dependent diabetics. I. PLT colonies detecting Dw10 and a new class II determinant distinct from HLA-D, DR, MB(DC), MT, and SB. Tissue Antigens 23: 290-300

9. NIAID Manual of Tissue Typing Techniques (1977) National Institutes of Health, Bethesda
10. Edwards AWF, Cavalli-Sforza LL (1965) A method for cluster analysis. Biometrics $21: 362-375$

11. Shaw S, Duquesnoy RJ, Smith PL (1981) Population studies of the HLA-linked SB antigens. Immunogenet 14: 153-162

12. Woolf $B$ (1955) On estimating the relation between blood group and disease. Ann Hum Genet 19: 251-253

13. Haldane JBS (1955) The estimation and significance of the logarithm of a ratio of frequencies. Ann Hum Genet 20: 309-311

14. Fisher RA (1960) The design of experiments, 7 th edn. Oliver and Boyd, Edinburgh, 1-248

15. Owerbach D, Lernmark $\AA$, Platz P, Ryder LP, Rask L, Peterson P, Ludvigsson J (1983) HLA-D region $\beta$-chain DNA endonuclease fragments differ between HLA-DR identical healthy and insulindependent diabetic individuals. Nature 303:815-817

16. Tait BD, Boyle A, Solty S, Cunningham T, Mandel T, Martin FIR, Doran T (1984) DR4 related antisera pattern differences in insulin-dependent diabetes mellitus and rheumatoid arthritis. Tissue Antigens 24: 228-233

17. Bach FH, Segall M, Rich S, Barbosa J (1982) HLA and susceptibility to type I diabetes. Tissue Antigens 20:28-32

18. Sheehy MJ, Rowe JR, MacDonald MJ (1985) A particular subset of HLA-DR4 accounts for all or most of the DR4 association in Type I diabetes. Diabetes 34: 942-944

19. Raum D, Awdeh Z, Yunis EJ, Alper CA, Gabbay KH (1984) Extended major histocompatibility complex haplotypes in Type I diabetes mellitus. J Clin Invest 74: 449-454

20. Platz P, Jakobsen B, Morling N, Ryder L, Svejgaard A, Thomsen M, Christy M, Kromann J, Benn J, Nerup J, Green A, Hauge M (1981) HLA-D and -DR antigens in genetic analysis of insulin-dependent diabetes mellitus. Diabetologia 21: 108-115

21. Dupont B, Braun DW Jr., Yunis EJ, Carpenter CB (1980) Joint report: HLA-D by cellular typing. In: Terasaki PI (ed) Histocompatibility Testing 1980, UCLA Tissue Typing Laboratory, Los Angeles, p 229-267

22. Termijtelen A, von den Berge AS, van Rood JJ (1983) LB-Q1 and LB-Q2: Two determinants defined in the primed lymphocyte test and independent of HLA-D/DR, MB/LB-E, or SB. Human Immunol 8:11-15

Received: 6 May, 1985

and in revised form: 18 September 1985

Dr. Michael Sheehy

American Red Cross Blood Services

4860 Sheboygan Avenue

Madison, WI 53705

USA 